PROCEEDINGS OF THE

AMERICAN MATHEMATICAL SOCIETY

Volume 125, Number 9, September 1997, Pages 2643-2648

S 0002-9939(97)03985-3

\title{
QUOTIENTS OF LITTLE LIPSCHITZ ALGEBRAS
}

\author{
NIK WEAVER \\ (Communicated by Palle E. T. Jorgensen)
}

\begin{abstract}
We prove a Tietze type theorem which provides extensions of little Lipschitz functions defined on closed subsets. As a consequence, we get that the quotient of any little Lipschitz algebra by any norm-closed ideal is another little Lipschitz algebra.
\end{abstract}

Let $X$ be a compact metric space. The Lipschitz algebra $\operatorname{Lip}(X)$ is the set of all scalar-valued Lipschitz functions on $X$ with the norm

$$
\|f\|_{L}=\max \left(\|f\|_{\infty}, L(f)\right),
$$

where

$$
L(f)=\sup _{\substack{x, y \in X \\ x \neq y}} \frac{|f(x)-f(y)|}{d(x, y)}
$$

is the Lipschitz number of $f$. The little Lipschitz algebra $\operatorname{lip}(X)$ is the subspace of $\operatorname{Lip}(X)$ consisting of those functions $f$ (called "little Lipschitz functions") with the property that for every $\epsilon>0$ there exists $\delta>0$ such that $d(x, y) \leq \delta$ implies $|f(x)-f(y)| \leq \epsilon \cdot d(x, y)$. It is well-known and easy to prove that $\operatorname{lip}(X)$ is a norm-closed subalgebra of $\operatorname{Lip}(X)$. Except where noted, we will assume the scalar field is real.

Every weak*-closed subalgebra of a Lipschitz algebra is isometrically isomorphic to another Lipschitz algebra ([9], Corollary B), as is the quotient of a Lipschitz algebra by any weak*-closed ideal ([9], Corollary C). A complex version of the first result also exists; it requires the subalgebra to be self-adjoint as well as weak*closed. Weak*-closed ideals are automatically self-adjoint, however the result on quotients is only valid up to isomorphism, not isometric isomorphism, in the complex case.

The analogous results for little Lipschitz algebras are as follows. Every normclosed subalgebra of a little Lipschitz algebra is isometrically isomorphic to another little Lipschitz algebra ([10], Theorem 3.4). It is also known that every norm-closed ideal of $\operatorname{lip}(X)$ is of the form

$$
I(K)=\left\{f \in \operatorname{lip}(X):\left.f\right|_{K}=0\right\}
$$

for some closed subset $K \subset X$ ([8], Corollary 4.3). This strongly suggests that the quotient of a little Lipschitz algebra by any norm-closed ideal will be isometrically isomorphic to another little Lipschitz algebra; indeed, it turns out that showing

Received by the editors March 18, 1996.

1991 Mathematics Subject Classification. Primary 46E15; Secondary 46J10, 46J15.

This research was supported by NSF grant DMS-9424370. 
$\operatorname{lip}(X) / I(K) \cong \operatorname{lip}(K)$ easily reduces to showing that every little Lipschitz function on $K$ extends with arbitrarily small increase in norm to a little Lipschitz function on $X$. However, this is by no means as trivial as the corresponding result for ordinary Lipschitz functions ([6], Theorem 1). It is the purpose of this note to supply the desired extension theorem. This answers a question raised in [11].

\section{The SEPARATION PROPERTY}

Some care is needed in formulating a theorem about extension of little Lipschitz functions. On the unit interval with the usual metric, although the set of Lipschitz functions is large there are no little Lipschitz functions besides the constant functions. Thus, in general it is not even possible to extend a function defined on a finite set. (Of course, every function on a finite metric space is little Lipschitz.) We need some extra condition on $X$; the appropriate condition is given in the following theorem.

Theorem 1. Let $X$ be a compact metric space. The following are equivalent:

(a) there exists $k>1$ such that for every $x, y \in X$ we have $f(x)=0$ and $f(y)=$ $d(x, y)$ for some $f \in \operatorname{lip}(X)$ with $L(f) \leq k$;

(b) for every $k>1$ and $x, y \in X$ we have $f(x)=0$ and $f(y)=d(x, y)$ for some $f \in \operatorname{lip}(X)$ with $L(f) \leq k$;

(c) there exists $k>1$ such that for every finite set $A \subset X$ and every $f \in \operatorname{Lip}(A)$, there is an extension $\tilde{f} \in \operatorname{lip}(X)$ of $f$ with $L(\tilde{f}) \leq k \cdot L(f)$;

(d) for every $k>1$, finite set $A \subset X$, and $f \in \operatorname{Lip}(A)$ there is an extension $\tilde{f} \in \operatorname{lip}(X)$ of $f$ with $L(\tilde{f}) \leq k \cdot L(f)$;

(e) $\operatorname{lip}(X, \mathbf{R})^{* *}$ is naturally isometrically isomorphic to $\operatorname{Lip}(X, \mathbf{R})$; and

(f) $\operatorname{lip}(X, \mathbf{C})^{* *}$ is naturally isometrically isomorphic to $\operatorname{Lip}(X, \mathbf{C})$.

Proof. (a) $\Rightarrow$ (c) is ([10], Proposition 1.3); (c) $\Rightarrow$ (f) is essentially ([1], Theorem $3.5)$; (f) $\Rightarrow$ (e) is trivial; (e) $\Rightarrow(\mathrm{d})$ is ([3], Theorem 1); and (d) $\Rightarrow(\mathrm{b}) \Rightarrow$ (a) is trivial.

A more complicated local condition, also equivalent to the above, was given in ([4], Theorem 1); see $([10], \S 1)$ for the proof of equivalence. We say that $X$ has the separation property if it satisfies any of the equivalent conditions of Theorem 1. For instance, every compact metric space whose metric is of the form $d^{\alpha}$ for some metric $d$ and $0<\alpha<1$ has the separation property ([1], Lemma 3.3). Also, every finite metric space has the separation property, as does the Cantor set with metric inherited from $[0,1]$. (It is possible to describe a "fat" Cantor set which does not have the separation property, but for which $\operatorname{lip}(X)$ does separate points. In other words, the separation by little Lipschitz functions is not uniform.)

It follows from ([10], Theorem 3.4) that for any compact metric space $X$ there is a compact metric space $Y$ with the separation property such that $\operatorname{lip}(X)$ is isometrically isomorphic to $\operatorname{lip}(Y)$.

\section{Fundamental lemmas}

Lemma 2. Let $X$ be a compact metric space with the separation property. Suppose $a_{n} \rightarrow a$ and $b_{n} \rightarrow b$ are convergent sequences in $X$ and let $K=\left\{a, a_{1}, a_{2}, \ldots, b, b_{1}\right.$, $\left.b_{2}, \ldots\right\}$. Then for every $f \in \operatorname{lip}(K)$ with $\|f\|_{L}<1$ there exists an extension $\tilde{f} \in \operatorname{lip}(X)$ of $f$ such that $\|\tilde{f}\|_{L}<1$. 
Proof. Let $\epsilon>0$ and $f \in \operatorname{lip}(K),\|f\|_{L}<1$. Choose $1 \geq \delta>0$ such that $d(x, y) \leq \delta$ implies $|f(x)-f(y)| \leq \epsilon \cdot d(x, y)$. Then choose $N \in \mathbf{N}$ such that $n>N$ implies $d\left(a_{n}, a\right), d\left(b_{n}, b\right) \leq \delta \epsilon / 2$. Let $A=\left\{a, a_{1}, \ldots, a_{N}, b, b_{1}, \ldots, b_{N}\right\}$ and by Theorem 1 (d) find $g \in \operatorname{lip}(X)$ such that $\left.g\right|_{A}=\left.f\right|_{A}$ and $L(g)<1$.

Let $\alpha=f(a)$ and $\beta=f(b)$ and let $p: \mathbf{R} \rightarrow \mathbf{R}$ be a piecewise linear continuous function with slope 0 on

$$
[\alpha-\delta \epsilon / 2, \alpha+\delta \epsilon / 2] \cup[\beta-\delta \epsilon / 2, \beta+\delta \epsilon / 2]
$$

and slope 1 elsewhere. We may arrange that $\left\|\mathrm{id}_{\mathbf{R}}-p\right\|_{\infty} \leq 2 \delta \epsilon$ where $\mathrm{id}_{\mathbf{R}}$ is the identity function on $\mathbf{R}, \operatorname{id}_{\mathbf{R}}(t)=t$.

Let $C=\|f\|_{\infty}$ and define $F=((p \circ f) \wedge-C) \vee C$ and $G=((p \circ g) \wedge-C) \vee C$. Note that these belong to $\operatorname{lip}(K)$ and $\operatorname{lip}(X)$ respectively. (In general, $\operatorname{lip}(X)$ is stable under finite lattice operations and composition with functions in $\operatorname{Lip}(\mathbf{R})$.) Also $L(G) \leq L(p) \cdot L(g)<1$ and $\|G\|_{\infty} \leq C<1$, so $\|G\|_{L}<1$.

Now observe that if $x=a_{n}$ with $n>N$ then $d(x, a) \leq \delta \epsilon / 2$, hence

$$
|f(x)-\alpha|=|f(x)-f(a)| \leq \delta \epsilon / 2 \quad \text { and } \quad|g(x)-\alpha|=|g(x)-g(a)| \leq \delta \epsilon / 2 .
$$

It follows that $p(f(x))=p(g(x))$, hence $F(x)=G(x)$. A similar argument shows that $F(x)=G(x)$ for $x=b_{n}$ with $n>N$, so $F$ and $G$ agree on $K-A$. But $f$ and $g$ agree on $A$, so we conclude that $F=\left.G\right|_{K}$. We will now use this fact to estimate $\left\|f-\left.G\right|_{K}\right\|_{L}$.

First observe that

$$
\left\|f-\left.G\right|_{K}\right\|_{\infty}=\|f-F\|_{\infty} \leq\left\|\operatorname{id}_{\mathbf{R}}-p\right\|_{\infty} \leq 2 \delta \epsilon \leq 2 \epsilon .
$$

To estimate $L\left(f-\left.G\right|_{K}\right)=L(f-F)$, let $x, y \in K$ with $d(x, y) \leq \delta$; then our choice of $\delta$ implies

$$
\begin{aligned}
|(f-F)(x)-(f-F)(y)| & \leq|f(x)-f(y)|+|F(x)-F(y)| \\
& \leq 2|f(x)-f(y)| \\
& \leq 2 \epsilon \cdot d(x, y) .
\end{aligned}
$$

On the other hand, if $x, y \in K$ satisfy $d(x, y) \geq \delta$ then

$$
\begin{aligned}
|(f-F)(x)-(f-F)(y)| & \leq|(f-F)(x)|+|(f-F)(y)| \\
& \leq 2 \delta \epsilon+2 \delta \epsilon \\
& \leq 4 \epsilon \cdot d(x, y) .
\end{aligned}
$$

Thus, we have $L(f-F) \leq 4 \epsilon$ and we conclude that

$$
\left\|f-\left.G\right|_{K}\right\|_{L}=\|f-F\|_{L} \leq 4 \epsilon .
$$

We have shown that for every $f \in \operatorname{lip}(K)$ with $\|f\|_{L}<1$ and every $\epsilon>0$ there exists $G \in \operatorname{lip}(X)$ with $\|G\|_{L}<1$ and $\left\|f-\left.G\right|_{K}\right\|_{L} \leq 4 \epsilon$. The lemma now follows by applying ([7], Theorem 4.13 (b) $\Rightarrow(\mathrm{c}))$ to the restriction map from $\operatorname{lip}(X)$ to $\operatorname{lip}(K)$.

We say that a function $f \in \operatorname{lip}(X)$ is $(\delta, \epsilon)$-expansive if $d(x, y) \leq \delta$ implies $|f(x)-f(y)| \leq \epsilon \cdot d(x, y)$. Thus, a function is little Lipschitz if and only if for every $\epsilon$ it is $(\delta, \epsilon)$-expansive for some $\delta$. 
Lemma 3. Let $X$ be a compact metric space with the separation property, let $K$ be a closed subset of $X$, and let $f \in \operatorname{lip}(K),\|f\|_{L}<1$. Then for every $\epsilon>0$ there exists $\delta>0$ with the following property: for every finite set $A \subset K$ there exists a $(\delta, \epsilon)$-expansive function $g \in \operatorname{lip}(X)$ such that $\left.g\right|_{A}=\left.f\right|_{A}$ and $\|g\|_{L}<1$.

Proof. By using the lattice operations in $\operatorname{lip}(X)$ it is enough to prove the weaker statement involving only two-element sets $A=\{x, y\} \subset K$. For if $\left\|g_{i j}\right\|_{L}<1$ and $g_{i j}$ agrees with $f$ on $\left\{x_{i}, x_{j}\right\}$ for each $1 \leq i \neq j \leq n$, then the function

$$
g=\bigvee_{i} \bigwedge_{j \neq i} g_{i j}
$$

has Lipschitz norm $<1$ and agrees with $f$ on $\left\{x_{1}, \ldots, x_{n}\right\}$. Furthermore, if each $g_{i j}$ is $(\delta, \epsilon)$-expansive then so is $g$.

Suppose then that this two-element version of the lemma fails for some $\epsilon>0$. Then for each $n \in \mathbf{N}$ let $\left\{a_{n}, b_{n}\right\} \subset K$ be a set on which $f$ agrees with no $(1 / n, \epsilon)$ expansive function $g \in \operatorname{lip}(X)$ with $\|g\|_{L}<1$. Choose a subsequence so that $\left(a_{n_{k}}\right)$ and $\left(b_{n_{k}}\right)$ both converge in $K$; invoking Lemma 2 , we then get a function $g \in \operatorname{lip}(X)$ with $\|g\|_{L}<1$ which agrees with $f$ on each pair $\left\{a_{n_{k}}, b_{n_{k}}\right\}$. But $g$ must be $(\delta, \epsilon)$ expansive for some $\delta>0$ since $g \in \operatorname{lip}(X)$. This is a contradiction and establishes the lemma.

An alternative proof of Lemma 3 goes by choosing $g \in \operatorname{lip}(X)$ which agrees with $f$ on $\{a, b\}$ where $a_{n_{k}} \rightarrow a, b_{n_{k}} \rightarrow b$, then modifying $g$ to get a function in $\operatorname{lip}(X)$ which agrees with $f$ on some $\left\{a_{n_{k}}, b_{n_{k}}\right\}$ and contradicts the choice of this pair. However, this argument will not work if $f(a)=f(b)$, in particular if $a=b$. In that case one still needs a Lemma 2 type argument.

\section{EXTENSIONS AND QUOTIENTS}

Theorem 4. Let $X$ be a compact metric space with the separation property and let $K \subset X$ be a closed subset. Then any $f \in \operatorname{lip}(K)$ with $\|f\|_{L}<1$ has an extension $\tilde{f} \in \operatorname{lip}(X)$ with $\|\tilde{f}\|_{L}<1$.

Proof. Let $\epsilon>0$ and $f \in \operatorname{lip}(K),\|f\|_{L}<1$. We will find $g \in \operatorname{lip}(K)$ with $\|g\|_{L}<1$ and $\left\|f-\left.g\right|_{K}\right\|_{L} \leq 4 \epsilon$. As in the proof of Lemma 2, this is enough; ([7], Theorem $4.13(\mathrm{~b}) \Rightarrow(\mathrm{c}))$ will then imply the desired conclusion.

By Lemma 3 find $\delta^{\prime}>0$ so that the restriction of $f$ to any finite subset of $K$ extends to a $\left(\delta^{\prime}, \epsilon\right)$-expansive function on $X$. Note that this implies that $f$ must itself be $\left(\delta^{\prime}, \epsilon\right)$-expansive. Define $\delta=\min \left(\epsilon, \delta^{\prime}\right)$ and find a finite $\delta$-net $A \subset K$. Let $g \in \operatorname{lip}(X)$ be a $(\delta, \epsilon)$-expansive function which satisfies $\|g\|_{L}<1$ and $\left.g\right|_{A}=\left.f\right|_{A}$.

We estimate $\left\|f-\left.g\right|_{K}\right\|_{\infty}$. For any $x \in K$ there exists $a \in A$ with $d(x, a) \leq \delta$. So

$$
\begin{aligned}
|f(x)-g(x)| & \leq|f(x)-f(a)|+|g(x)-g(a)| \\
& \leq \delta+\delta \\
& \leq 2 \epsilon .
\end{aligned}
$$

Thus $\left\|f-\left.g\right|_{K}\right\|_{\infty} \leq 2 \epsilon$.

We estimate $L\left(f-\left.g\right|_{K}\right)$. For any $x, y \in K$ with $d(x, y) \leq \delta$, we have

$$
\begin{aligned}
\left|\left(f-\left.g\right|_{K}\right)(x)-\left(f-\left.g\right|_{K}\right)(y)\right| & \leq|f(x)-f(y)|+|g(x)-g(y)| \\
& \leq 2 \epsilon \cdot d(x, y)
\end{aligned}
$$


since $f$ and $g$ are both $(\delta, \epsilon)$-expansive. On the other hand, if $x, y \in K$ satisfy $d(x, y) \geq \delta$ then find $a, b \in A$ with $d(x, a), d(y, b) \leq \delta$; we then have

$$
|f(x)-g(x)| \leq|f(x)-f(a)|+|g(x)-g(a)| \leq 2 \epsilon \cdot d(x, a) \leq 2 \delta \epsilon
$$

and likewise $|f(y)-g(y)| \leq 2 \delta \epsilon$. Hence

$$
\begin{aligned}
\left|\left(f-\left.g\right|_{K}\right)(x)-\left(f-\left.g\right|_{K}\right)(y)\right| & \leq|f(x)-g(x)|+|f(y)-g(y)| \\
& \leq 4 \delta \epsilon \\
& \leq 4 \epsilon \cdot d(x, y) .
\end{aligned}
$$

We conclude that $L\left(f-\left.g\right|_{K}\right) \leq 4 \epsilon$, so that $\left\|f-\left.g\right|_{K}\right\|_{L} \leq 4 \epsilon$ as desired.

The idea of using an approximation lemma argument to prove an extension theorem comes from [2].

Corollary 5. Let $X$ be a compact metric space and let $I$ be a norm-closed ideal of $\operatorname{lip}(X)$. Then $\operatorname{lip}(X) / I$ is isometrically isomorphic to $\operatorname{lip}(Y)$ for some compact metric space $Y$. If $X$ has the separation property then we may take $Y=K$ where $I=I(K)$.

Proof. It follows from ([10], Theorem 3.4) that $\operatorname{lip}(X)$ is isometrically isomorphic to $\operatorname{lip}\left(X^{\prime}\right)$ for some compact metric space $X^{\prime}$ with the separation property. Thus, without loss of generality we may assume that $X$ has the separation property.

By ([8], Corollary 4.3) $I$ is of the form $I=I(K)$, the ideal of all functions which vanish on some closed subset $K \subset X$. Now consider the restriction map $T: \operatorname{lip}(X) \rightarrow \operatorname{lip}(K)$. It is nonexpansive, it is onto by Theorem 4 , and its kernel is clearly $I$. Also, according to Theorem 4 , for any $f \in \operatorname{lip}(K)$ and $\epsilon>0$ there exists $\tilde{f} \in T^{-1}(f)$ with $\|\tilde{f}\|_{L} \leq\|f\|_{L}+\epsilon$. This shows that $T$ defines an isometry between $\operatorname{lip}(X) / I$ and $\operatorname{lip}(K)$.

Complex versions of Theorem 4 and Corollary 5 follow from the real versions by separating into real and imaginary parts. This introduces a factor of $\sqrt{2}$ in the extension of a little Lipschitz function, and the isometric isomorphism of Corollary 5 becomes a mere isomorphism. In fact, extension of complex-valued Lipschitz functions cannot be achieved with arbitrarily small increase in Lipschitz number $([5], \S 3)$. The same is true of little Lipschitz functions and the same example shows this. Namely let $X=\left\{x_{0}, x_{1}, x_{2}, x\right\}$ with $d\left(x_{m}, x_{n}\right)=1(m \neq n)$ and $d\left(x_{m}, x\right)=1 / 2$. Let $K=\left\{x_{0}, x_{1}, x_{2}\right\}$ and define $f: K \rightarrow \mathbf{C}$ by $f\left(x_{m}\right)=e^{2 \pi i m / 3}$. Then $L(f)=\sqrt{3}$ but the Lipschitz number of any extension to $X$ is at least 2 . As we are dealing with finite sets, every function is little Lipschitz and so this example falsifies the exact complex version of Theorem 4. It also shows that isometric isomorphism is not possible in the complex version of Corollary 5.

Theorem 4 is also sharp in the following sense. While the Lipschitz number of the extension can be brought arbitrarily close to the Lipschitz number of $f$, in general one cannot have $L(\tilde{f})=L(f)$. To see this take $X=\{1 / n: n \in \mathbf{N}\} \cup\{0\}$ with metric inherited from $[0,1]$ and define $f(0)=0, f(1)=1$ on $K=\{0,1\}$. Then $L(f)=1$ and it is easy to see that there is only one extension of $f$ to $X$ with Lipschitz number 1 , namely the function $\tilde{f}(t)=t$. But this function is not little Lipschitz. Note that $X$ does have the separation property. 


\section{REFERENCES}

1. W. G. Bade, P. C. Curtis, and H. G. Dales, Amenability and weak amenability for Beurling and Lipschitz algebras, Proc. Lond. Math. Soc. 55 (1987), 359-377. MR 88f:46098

2. S. Grabiner, The Tietze extension theorem and the open mapping theorem, Amer. Math. Monthly 93 (1986), 190-191. MR 88a:54034

3. L. G. Hanin, Kantorovich-Rubenstein norm and its application in the theory of Lipschitz spaces, Proc. Amer. Math. Soc. 115 (1992), 345-352. MR 92i:46026

4. L. I. Hedberg, The Stone-Weierstrass theorem in Lipschitz algebras, Ark. Mat. 8 (1969), 63-72. MR 41:5973

5. T. M. Jenkins, Banach Spaces of Lipschitz Functions on an Abstract Metric Space, Ph.D. Thesis, Yale University (1968).

6. E. J. McShane, Extension of range of functions, Bull. Amer. Math. Soc. 40 (1934), 837-842.

7. W. Rudin, Functional Analysis, (second edition), McGraw-Hill, 1991. MR 92k:46001

8. D. R. Sherbert, The structure of ideals and point derivations in Banach algebras of Lipschitz functions, Trans. Amer. Math. Soc. 111 (1964), 240-272. MR 28:4385

9. N. Weaver, Order completeness in Lipschitz algebras, J. Funct. Anal. 130 (1995), 118-130. MR 96f: 46048

10. _ Subalgebras of little Lipschitz algebras, Pac. J. Math. 173 (1996), 283-293.

11. Lipschitz algebras and derivations of von Neumann algebras, J. Funct. Anal. 139 (1996), 261-300.

Department of Mathematics, University of California at Los Angeles, Los Angeles, CALIFORNIA 90024

E-mail address: nweaver@math.ucla.edu 